Muséologies

Les cahiers d'études supérieures

muséologies

\title{
Du musée ethnologique au musée multiculturel : bilan et enjeux d'une rénovation institutionnelle européenne
}

\section{Fabien Van Geert}

Volume 8, numéro 2, 2016

Les nouveaux paradigmes

URI : https://id.erudit.org/iderudit/1050762ar

DOI : https://doi.org/10.7202/1050762ar

Aller au sommaire du numéro

Éditeur(s)

Association Québécoise de Promotion des Recherches Étudiantes en

Muséologie (AQPREM)

ISSN

1718-5181 (imprimé)

1929-7815 (numérique)

Découvrir la revue

Citer cet article

Van Geert, F. (2016). Du musée ethnologique au musée multiculturel : bilan et enjeux d'une rénovation institutionnelle européenne. Muséologies, 8(2),

93-104. https://doi.org/10.7202/1050762ar

Tous droits réservés (C Association Québécoise de Promotion des Recherches Étudiantes en Muséologie (AQPREM), 2018
Ce document est protégé par la loi sur le droit d'auteur. L’utilisation des services d'Érudit (y compris la reproduction) est assujettie à sa politique d'utilisation que vous pouvez consulter en ligne.

https://apropos.erudit.org/fr/usagers/politique-dutilisation/ 
Article deux

Du musée ethnologique au musée multiculturel: bilan et enjeux d'une rénovation institutionnelle européenne Fabien Van Geert 
Fabien Van Geert est docteur en gestion de la culture et du patrimoine de l'Université de Barcelone après avoir réalisé une thèse portant sur l'influence du multiculturalisme dans la rénovation des musées ethnologiques. Depuis 2017, il est Maître de conférences en muséologie à l'Université Paris 3-Sorbonne Nouvelle. 


\section{Un nouveau sens pour les institutions ethnologiques}

Les musées nationaux occidentaux ont contribué, depuis leur création au $19^{\mathrm{e}}$ siècle, à la formation d'une identité nationale par la mise en exposition d'une histoire et d'un patrimoine communs ${ }^{1}$. En tant que lieux de diffusion de valeurs, symboliquement et culturellement construits ${ }^{2}$, ils y créent une mémoire et des significations au travers d'un processus de sélection d'éléments définis par des intérêts politiques ${ }^{3}$. Ainsi, alors qu'au cours du $19^{\mathrm{e}}$ siècle, leur rôle était d'inculquer un sens moral à la population, dès la fin du $20^{\mathrm{e}}$ siècle, le rôle des musées est progressivement devenu celui d'encourager l'acceptation de la diversité culturelle. Dans un contexte de débats quant à une reconnaissance de la nature plurielle des sociétés en tant que garant démocratique des libertés ${ }^{4}$, il fut en effet considéré que ces institutions possédaient un rôle majeur dans la construction d'une nouvelle identité multiculturelle, inclusive et tolérante envers la diversité culturelle ${ }^{5}$. Dans ce contexte, les musées ethnologiques et coloniaux européens, jadis liés à la consolidation de la discipline anthropologique dans un contexte d'expansion coloniale, trouvèrent une possibilité de se réinventer, alors que leurs collections et leurs histoires ont toujours été intrinsèquement liées aux relations entre l'Europe et le monde'.

1 MACDONALD, Sharon. " Theorizing museums: an introduction ". MACDONALD, Sharon et FYFE, Gordon (dir.). Theorizing museums. Representing identities and diversity in a changing world. Oxford: Blackwell, 1996, p. 9.

2 BENNETT, Tony. The birth of the museum. London: Routledge, 1995, p. 68.

3 SILVEN, Eva. «Difficult matters. Museums, materiality, multivocality ". SVANBERG, Fredrick (dir.). The Museum as Forum and Actor. Stockholm: Statens historiska museum, 2010, p. 140.

4 TAYLOR, Charles. Multiculturalisme. Différence et démocratie. Paris: Champs essais, 1994 (1992), p. 13. 5 WATSON, Sheila. "Museums and their Communities". WATSON, Sheila (dir.). Museums and their Communities. London: Routledge, 2007, p. 6.

6 Voir MAZE, Camille, POULARD, Frédéric et Christelle VENTURA (dir.). Les Musées d'ethnologie. Culture, politique et changement institutionnel. Paris : Éditions du Comité des travaux historiques et scientifiques, 2013.
Depuis les années 1960, ces institutions étaient en effet plongées dans une profonde crise existentielle. Le désintérêt des collections matérielles au sein du développement des nouvelles méthodes anthropologiques, tant structuralistes que symboliques, fit perdre le rôle scientifique de ces institutions. Cet " âge universitaire de l'Anthropologie ${ }^{7}$ ", apparu dès les années 1960, consomma ainsi plus ou moins définitivement en Europe le divorce entre le musée et les anthropologues, ces derniers se tournant vers l'université en tant que lieu de recherche. Parallèlement, à la suite de la décolonisation et de la critique faite à l'anthropologie pour son rôle de justification scientifique des rapports de pouvoir coloniaux ${ }^{8}$, les musées ethnologiques ne rencontrèrent plus les intérêts géopolitiques du nouvel ordre mondial, marqué par une reformulation des identités postcoloniales. Cet "âge de la déconstruction" remit en effet en cause les standards traditionnels de la muséologie, alors que la " question ethnique ", liée aux revendications sociales et culturelles de nombreux secteurs de la population, a touché de plein fouet les institutions nationales et ethnologiques ${ }^{9}$. Les principales critiques concernèrent tout particulièrement les manières de représenter les cultures ${ }^{10}$, en remettant en cause les perspectives européocentristes et coloniales de représentation ${ }^{11}$, en redéfinissant de nouveaux liens entre les

7 STURTEVANT, William C. « Does Anthropology need museums? ", Proceedings of the Biological Society of Washington. $\mathrm{n}^{\circ} 82,1969$, p. 624.

8 Dans le contexte français, voir LECLERC, Gérard (dir.). Anthropologie et colonialisme. Paris: Fayard, 1972. Pour la Grande-Bretagne, voir ASAD, Talal (ed.). Anthropology and the Colonial Encounter. London: Ithaca Press, 1973.

9 AMES, Michael M. Cannibal Tour and Glass Boxes. The Anthropology of Museums. Vancouver: UBC Press, 1992, p. 152.

10 LAVINE, Steven D. "Culture and Representation ". LAVINE, Steven D. et Ivan KARP (dir.). Exhibiting cultures. The Poetics and Politics of Museum Display. Washington: Smithsonian Institution Press, 1991, p. 11.

11 BARRINGER, Tim et Tom FLYNN. «Introduction ". BARRINGER, Tim et Tom FLYNN (ed.). Colonialism and the Object. Empire, Material Culture and the Museum. Abingdon/ New York: Routledge, 1998, p. 1. 
musées et ces populations ${ }^{12}$, et en s'interrogeant sur la possession et la propriété des collections ethnographiques ${ }^{13}$.

Influencés par ces réflexions, tout particulièrement développées en Amérique du Nord et en Océanie, de profonds débats s'engagèrent dans les musées européens, au cours des années 1990, quant au rôle de ces institutions dans la création du " vivre ensemble ${ }^{14}$ ". Ces derniers tentèrent ainsi de se convertir en partenaires et médiateurs privilégiés dans la promotion $\mathrm{du}$ " dialogue des cultures " et du respect des diversités ${ }^{15}$, tels que promus par les institutions internationales en tant que garant de la cohésion sociale. Dès les années 1990, et tout au long des années 2000, s'inaugurèrent notamment à Paris, Bâle, Francfort, Genève, Berlin ou Anvers, de grandes rénovations rompant plus ou moins nettement avec leurs ancêtres coloniaux, au sein de nouveaux édifices dessinés par les architectes-stars du moment.

Plus d'une décennie après le début de cette vague de rénovations qui s'est étendue à l'ensemble du continent européen, et dont certaines sont encore actuellement en cours, il convient de poser un bilan sur ces transformations. Pour ce faire, cet article se propose d'analyser ce processus et ses différentes "pratiques expographiques ${ }^{16}$ ", au travers d'une approche critique de l'institution muséale, et tout particulièrement de sa représentation du multiculturalisme. Dans un premier temps, il tentera une théorisation des bases muséologiques ainsi que des nouvelles méthodologies

12 SIMPSON, Moira G. Making representations. Museums in the Post-Colonial Era. London: Routledge, 1996, p. 3. 13 STOCKING, George W. "Essays on Museums and Material Culture ". STOCKING, George W. (ed.). Objects and Others: Essays on Museums and Material Culture. Madison: The University of Wisconsin Press, 1985, p. 11.

14 Dans le cas britannique, voir notamment HALL, Stuart. "Culture, Community, Nation ". Cultural Studies. vol. 7, $\mathrm{n}^{\circ}$ 3, 2003. Dans le cas français, voir notamment TOURAINE, Alain. Pouvons-nous vivre ensemble? Égaux et différents. Paris: Fayard, 1997; ou WIEVIORKA, Michel. Une société fragmentée? Le multiculturalisme en débat. Paris : La découverte, 1997. 15 BOUTTIAUX, Anne-Marie. Musées d'Ethnographie et Cultures du Monde. International Network of Ethnography Museums and World Cultures. Tervuren: Musée Royal de l'Afrique Centrale, 2008, p. 21. mises en place au sein de ces rénovations. Dans un deuxième temps, l'article se penchera sur un cas concret de rénovation, le Musée de la culture mondiale de Göteborg, analysé en 2012 au cours d'un travail de terrain, qui constitue sans aucun doute le paroxysme de ce type de rénovation censée promouvoir des valeurs cosmopolites au sein de la population. L'analyse de cette institution, des stratégies mises en place, ainsi que de sa méthodologie permettra d'en faire émerger l'idéologie sousjacente tout en caractérisant, à partir de cet exemple, quelques-uns des principaux défis auxquels ces rénovations se trouvent actuellement confrontées.

\section{Stratégies et méthodologies du musée multiculturel}

Bien que les rénovations des musées ethnologiques soient très différentes entre elles ${ }^{17}$, il est possible de définir une série de points communs. D’un point de vue muséologique, le principal serait la mise en place de stratégies visant à déconstruire les discours coloniaux jadis développés par les ancêtres de ces musées, en s'ouvrant à de nouvelles dynamiques d'acquisition de collections. Cette perspective correspondrait à trois grandes stratégies, non exclusives les unes des autres, parfois mises au point au sein d'une même institution, voire d'une même exposition, et pouvant être rapprochées de la muséologie critique ${ }^{18}$. Au sein de ces dernières, les musées réactiveront en effet les réflexions liées à la nouvelle

16 MAIRESSE, François et Cécilia HURLEY. "Éléments d'expologie: matériaux pour une théorie du dispositif muséal ". MediaTropes eJournal. vol. 3, n 2, 2012, p. 3. 17 Pour une synthèse approfondie de ces stratégies, voir VAN GEERT, Fabien. "The Multicultural Pill and its Museological Effects for the Recovery of the European Ethnological Museums ". Museological Review, n 19, 2015, p. 45-53.

18 LORENTE, Jesús P. "Critical museology: Museums and exhibitions as public spaces of controversy and collective participation ". Art.es. no 52, 2012, p. 101-102. 
muséologie, quant à leur rôle dans la société, " au service de l'homme et de son développement ${ }^{19}$ ", ainsi qu'à leur volonté de se convertir en " forum » de la diversité culturelle, en tant que lieu de confrontation, d'expériences et de débats ${ }^{20}$.

La première stratégie consiste en la présentation de l'esthétique des collections afin d'insister sur la diversité des conceptions formelles et de mettre en valeur la complexité culturelle des groupes les ayant produites. Plus proche des musées et galeries d'art que de l'anthropologie de l'art telle que conçue par Franz Boas dès le début du $20^{\mathrm{e}}$ siècle ou par le Musée de l'Homme parisien dans sa tentative de jeter des ponts entre l'approche culturelle et esthétique des collections ${ }^{21}$, cette stratégie ne sera pas exempte de critiques de la part des anthropologues. Ces dernières portèrent surtout sur son approche esthétique ethnocentriste ${ }^{22}$, qui privilégie la présentation des artefacts de manière séparée, à partir de leur " aura " esthétique définie par des critères occidentaux, plutôt que pour leur rôle historique ou de témoins d'anciennes cultures. Alors que cette stratégie est depuis amplement employée dans la rénovation des musées ethnologiques, Wastiau alerta d'une possible «menace de l'esthétisme planant sur les musées ethnologiques ${ }^{23}$ ".

La deuxième stratégie, que l'on pourrait qualifier de "sociétale », consiste en la création de " forums de la diversité culturelle " au sein des musées, souvent rebaptisés pour l'occasion "musées des cultures du monde", en intégrant souvent plus ou moins explicitement la question de l'immigration en Europe. Particulièrement portées sur le contemporain ainsi que sur les contacts entre cultures, ces rénovations constitueraient l'expression muséale des théories anthropologiques et sociologiques sur les sociétés postcoloniales, transnationales et globalisées, définies par des auteurs tels qu'Appadurai ${ }^{24}$, Hannerz ${ }^{25}$ ou Bauman $^{26}$. Les musées présentent ainsi leurs collections de manière thématique, et non plus par zones géographiques ou par groupes de populations, en présentant ensemble des collections issues de cadres spatio-temporels différents. Ils s'ouvrent en outre vers de nouveaux " objets-témoins ", " métissés ${ }^{27}$ ", produits de "l'indigénisation de la modernité ${ }^{28}$ ", issus de la culture populaire, mais également vers des exemples d'art contemporain dit "global ", popularisé par des expositions de grande envergure tenues en Europe dès la fin des années 1980, dont "Les Magiciens de la Terre " au Centre George-Pompidou en 1989 constitua une première.

La troisième stratégie, réflexive, est particulièrement liée à une révision critique de l'histoire et des pratiques de ces institutions, menée tout particulièrement par les anthropologues se rapprochant alors des musées, en tant que sujets de réflexion et lieu de représentation ${ }^{29}$. Au travers d'expositions réalisées par le

24 Voir APPADURAI, Arjun. Modernity at Large. Cultural Dimensions of Globalization, Minneapolis : University of Minnesota Press, 1996.

25 Voir HANNERZ, Ulf. Transnational Connections. Culture, people, places. London/New York: Routledge, 1996. 26 Voir BAUMAN, Zygmunt. Globalization: The Human Consequences. Cambridge: Polity, 1998.

27 Voir TURGEON, Laurier. Patrimoines métissés. Contextes coloniaux et postcoloniaux, Québec/Paris: Presses de l'Université Laval/Éditions de la Maison des sciences de l'homme, 2003. 28 SAHLINS, Marshall. "Goodbye to Tristes Tropes: Ethnography in the context of modern world history ". BOROFSKY, Robert (ed.). Assessing Cultural Anthropology. New York: McGraw Hill, 1994, p. 271.

29 BOUQUET, Mary. "Introduction. Academic Anthropology and the Museums. Back to the future ". BOUQUET, Mary (ed.). Academic Anthropology and the Museums. Back to the future. New York/Oxford: Berghahn Books, 2001, p. 1.
22 Pour une analyse anthropologique de cette approch ethnocentrique, voir tout particulièrement PRICE Sally. Primitive Art in Civilized Places. Chicago : University of Chicago Press, 1989.

23 WASTIAU, Boris. "La reconversion du musée glouton ". GONSETH Marc-Olivier, HAINARD, Jacques et Roland KAEHR (dir.). Le Musée Cannibale. Neuchâtel: Musée d'Ethnographie, 2002, p. 105. 
musée, ou grâce à la participation d'artistes qui prennent le rôle de conservateurs en vue de défamiliariser les visiteurs avec les collections, selon une approche définie par Shelton de "muséologie praxiologique ${ }^{30}$ ", de nombreux musées vont proposer une perspective critique sur leurs pratiques expographiques. De véritables parcours muséologiques alternatifs vont se créer au sein des collections, des expositions, donnant parfois lieu à l'aménagement de " salles historiques " au sein de certains musées. D'autres pousseront la réflexion encore plus loin, en donnant lieu à de véritables " expositions d'auteur ", particulièrement réflexives dans leurs pratiques interprétatives. Les exemples paradigmatiques de ces expériences sont, dans le contexte britannique, les expositions menées par Shelton et ses élèves de muséologie critique au Musée de Brighton et au Musée Horniman de Londres au cours des années $1990^{31}$. Dans le monde francophone, c'est tout particulièrement le cas de l'expérience du Musée d'ethnographie de Neuchâtel, qui propose, depuis les années 1980, en lien avec l'Institut d'Ethnologie de l'Université de Neuchâtel, une " muséologie de la rupture ", visant à rompre la relation " sacralisante " existante entre l'objet et le visiteur au travers d'un regard décalé sur les objets se connotant par opposition plus que par rapprochements ${ }^{32}$.

Dans la mise en place de ces stratégies, ces rénovations créeront de nouvelles méthodologies en tentant d'intégrer de nouveaux points de vue dans l'interprétation des collections. À partir d'une approche " objet-sujet ", basée sur l'idée que les artefacts ne constituent pas seulement des substituts de certaines sociétés, mais qu'ils entretiennent plutôt des liens avec

30 SHELTON, Anthony. "Unsettling the meaning: critical museology, art and anthropological discourses $»$. BOUQUET, Mary (dir.). op.cit., p. 147.

31 Pour une description de ces expériences dans les musées anglais, voir SHELTON. op. cit.

32 DESVALLÉES, André et François MAIRESSE.

"La muséologie de la rupture ». DESVALLÉES, André et

François MAIRESSE (dir.). Dictionnaire encyclopédique de muséologie. Paris: Armand Colin, 2011, p. 635.

33 AMES. op.cit., p. 54.

34 SIMPSON, op.cit., p. 1. leurs descendants ${ }^{33}$, ces pratiques firent naître de profondes réflexions sur l'interprétation des collections, sur la classification et les valeurs attachées aux objets ainsi que sur les demandes d'autoreprésentation et d'auto-expression ${ }^{34}$. En effet, à partir de l'application aux musées de théories développées dans le monde éducatif par Pratt ${ }^{35}$, Clifford indiqua que les relations de pouvoir colonial se retrouvaient dans l'interprétation des collections faite par les musées occidentaux, et plus particulièrement par les institutions ethnologiques ${ }^{36}$. Afin de dépasser ces relations de pouvoir, Clifford appelait à la collaboration et au partage de l'autorité dans les musées à partir de la création d'une « zone de contacts ". Ce terme fut particulièrement intégré par les musées européens, voulus inclusifs, en tant que synonyme de " muséologie participative ", telle que définie par Crooke ${ }^{37}$, en tentant de laisser la place, au sein des médiations établies par le musée entre le public et les objets, aux membres des cultures représentées ainsi qu'à leurs descendants, compris en tant que « communautés sources ${ }^{38}$ ».

La principale complexité de cette pratique réside cependant dans le type de rapports établi entre le musée et les différents participants, en vue de trouver un équilibre qui permette une véritable collaboration. En effet, cette participation ne doit pas se limiter à ce que les participants confirment le discours du musée qu'ils auraient intégré inconsciemment, mais doit également tenter d'éviter une radicalisation de la critique de l'autorité muséale qui postulerait l'idée d'une " altérité absolue » ou d'un relativisme extrême propre aux postures postmodernes les plus déconstructivistes. Dans la mise en place de ce type de méthodologie,
35 Voir PRATT, Mary Louise. "Arts of the contact zone". Profession. $\mathrm{n}^{\circ}$ 91, 1991, p. 33-40.

36 CLIFFORD, James. Routes. Travel and Translation in the late twentieth century, Cambridge: Harvard University Press, 1997 , p. 188.

37 Voir CROOK, Elizabeth. "Museums \& Communities ". MACDONALD, Sharon (ed.). A companion to Museum Studies. Malden/Oxford: Blackwell, 2006.

38 PEERS, Laura et Alison K. BROWN. "Introduction".

PEERS, Laura et Alison K. BROWN (ed.). Museums and Sources Communities. London/ New York: Routledge, 2003, p. 1. 
les musées doivent ainsi réfléchir sur la place donnée aux " communautés sources", créées par le musée, et dont le discours sur les collections doit être complémentaire aux méthodes " scientifiques " de recherche de la connaissance. Simpson a en effet déjà rappelé le fait que les communautés sont souvent nostalgiques dans leurs représentations de leur passé ${ }^{39}$, courant le risque d'une « ethno-nostalgie $^{40}$ ", où, comme l'annonçait Crooke, " les nouvelles voix peuvent être aussi partiales que les anciennes ${ }^{41} "$.

\section{Le cas de la rénovation du Musée de Göteborg}

Ce rôle de l'institution ethnologique ainsi que ses différentes stratégies de déconstruction se retrouvent très clairement au sein du Musée de la culture mondiale (Världskulturmuseet) de Göteborg. En effet, parallèlement à l'entrée de la Suède dans l'Union Européenne en 1995, dont la conséquente immigration fut perçue comme symbole de sa nouvelle modernité ${ }^{42}$, l'État définit la promotion du dialogue interculturel comme l'un de ses principaux objectifs, parallèlement à la démocratisation culturelle $^{43}$. Depuis 1995, l'ensemble des musées suédois sont en effet obligés par mandat officiel d'aborder la nature multiculturelle du pays. Dans ce contexte, le parlement suédois, alors gouverné par les socio-démocrates, proposa, dès 1996, la transformation de l'ancien Musée d'ethnologie de Göteborg, créé en 1946 et fermé en 1999, en Musée de la culture mondiale (Världskulturmuseet). Cette dernière devait ainsi donner lieu à un " musée de pointe " du cosmopolitisme au sein de la création du

39 SIMPSON, op. cit., p. 68.

40 CHAUMIER, Serge. "L'identité, un concept embarrassant, constitutif de l'idée de musée ". Cultures et Musées. $n^{\circ} 6$, 2005, p. 26.

41 CROOKE. op. cit., p. 177-184.

42 LAGERVKVIST, Cajsa. "The Museum of World

Culture: a 'glocal' museum of a new kind ". GOODNOW, Katherine et Haci AKMAN (ed.). Scandinavian Museums and Cultural Diversity. London: Berghahn, 2008, p. 92. nouveau consortium national du Musée de la culture du monde (Världskulturmuseerna) regroupant les collections extra-européennes conservées au Musée d'ethnologie, au Musée des Antiquités de la Méditerranée et du Proche Orient, et au Musée des Antiquités de l'Extrême-Orient, tous trois situés à Stockholm. Il fut en effet affirmé du côté gouvernemental que « les musées à vocation ethnographique doivent, plus que jamais auparavant, remplir une mission importante dans la promotion des contacts entre les cultures suédoises et non-suédoises ${ }^{44}$ ".

À la suite de l'ouverture du nouveau musée de Göteborg en 2004 au sein d'un bâtiment à l'architecture moderniste flambant neuf, la jeune équipe " cosmopolite " du musée, interdisciplinaire et internationale, réalisa, sous la direction de Jette Sandahl, figure de proue de la muséologie scandinave, une série d'expositions temporaires de grand format. Ces dernières, produites en version bilingue suédois-anglais, se voulaient particulièrement novatrices et inclusives, visant à « reconnaître la diversité culturelle comme une richesse du patrimoine humain et du pluralisme culturel, comme condition de base pour la paix, l'harmonie et la stabilité culturelle dans le monde ${ }^{45}$ ". Pour ce faire, le musée se basa sur une volonté d'engagement démocratique et critique de l'institution dans les débats contemporains globaux et locaux, mélangeant au sein de ses expositions des témoignages, des objets de la culture de masse, de l'art contemporain ainsi que certains objets issus des collections de l'ancien musée d'ethnographie. En outre, le musée établit également, dès sa création, le projet "Mouseion " en vue de collaborer avec le centre d'Études
43 Pour une analyse détaillée des politiques culturelles suédoises, voir HARDING, Tobias. "Sweden. Council of Europe/ERCarts ". Compendium of Cultural Policies and Trends in Europe. $14{ }^{\mathrm{e}}$ édition, 2012. <http://www.culturalpolicies.net/web/sweden.php> (consulté en juillet 2016). 44 REGERING, Sveriges. Direktiv 1996:110. Organisationskommitté för etablering av ett världskulturmuseum i Göteborg. Stockholm: Kulturdepartementet, 1996, p. 142-143.

45 SANDAHL, Jette. "The Included Other-the Oxymoron of Contemporary Ethnographic Collections? ". Forum for Anthropology and Culture. $\mathrm{n}^{\circ}$ 4, 2008, p. 211. 
Globales de l'Université de Göteborg, sans pourtant arriver à le réaliser après l'ouverture du musée.

Les principales expositions de grands formats de l'institution abordèrent ainsi différentes thématiques sociales contemporaines à partir d'une perspective "glocale ", mondiale mais également suédoise, propre à la stratégie " sociétale " vue précédemment, qui eut un grand succès en termes d'attraction des jeunes publics, qui constituèrent plus de $60 \%$ de l'ensemble du public entre 2004 et $2008^{46}$. C'est le cas des religions ( $390 \mathrm{~m}^{3}$ Spirituality » en 2004-2006, “ Vodou " en 2009-2010), des épidémies ("No name fever. Aids in the age of globalization " en 2004-2006), du développement durable ("Fair Fashion », en 2007-2008), des luttes pour les droits de l'homme (notamment "Dishum Dishum. Fight the Tradition " et " Take Action! 83 ways to change the world " en 2008), de la culture contemporaine globalisée ( Bollywood » en 2009-2010 ou "Kimono Fusion " en 2010-2011), de la traite des personnes ("Trafficking » en 2006-2008), du genre ("Gender Blender » en 2006-2007, «Jerusalem » en 2010, « Secret Love " en 2012) ou encore des déplacements de population ("Destination X» en 2011-2013).

En outre, le musée illustra sa volonté de traitement postcolonial de son passé institutionnel au travers d'expositions. La première action de cette stratégie réflexive et déconstructiviste se fit au travers d'une pratique de " muséologie praxiologique ", en lien avec l'artiste Fred Wilson, particulièrement actif dans ce genre de pratique, au cours de l'exposition « Site Unseen: Dwellings of the Demons " (2004-2007). Cette dernière souhaitait interpréter l'inconscient du musée, en mettant en scène, à partir des collections de l'institution, sa réflexion sur la question de l'exclusion et de l'exposition des structures de pouvoir, de racisme et de sexisme ainsi que

46 Pour une analyse des publics du musée, voir ALIN, Margareta. So Far. Göteborg: The Museum of World Culture, 2009.

47 Voir MUÑOZ, Adriana. "La creación del Museo de la Cultura del Mundo, Gotemburgo (Suecia): tentativas de cambio de paradigma y prácticas museales ". Baukara. $\mathrm{n}^{\circ} 4$, 2013, p. 68-84. de l'absence de contextualisation historique des objets qui constitueraient les bases du musée. D'autres expositions présentèrent également ces propos du musée. Ce fut le cas de "Sister of Dreams " (2004-2008), dont le but était de déconstruire les stéréotypes sur le primitivisme, ou encore de "Traces of Congo " (2008) qui explorait le rôle, bien que modeste, joué par les pays nordiques, et principalement certains de ses citoyens, dans l'exploitation coloniale du Congo au cours du $19^{\mathrm{e}}$ siècle. Enfin, le musée explora une partie de son histoire coloniale, en traitant de la manière dont certaines œuvres sont arrivées au musée, s'engageant ainsi dans les débats et les polémiques autour des spoliations et des restitutions d'objets. Cette volonté donna lieu à l'exposition "A Stolen World " (2008-2011) qui présentait l'histoire de textiles péruviens arrivés au musée vers 1930 en provenance de la péninsule de Paracas, à la suite de probables pillages dans les tombes de la région. L'objectif de cette exposition était ainsi d'adresser la problématique de la possession du patrimoine, en lien avec le code de déontologie du Conseil international des musées (ICOM).

Cette réflexion se retrouve également tout particulièrement dans le travail réalisé sur les collections historiques du musée à partir de 2012, auxquelles le musée tourna dans un premier temps le dos, où la participation de différents collectifs, tant suédois qu'internationaux mais également institutionnels, permirent d'offrir de nouvelles interprétations sur les collections. Les résultats de ce type de «muséologie participative " donnèrent lieu en partie à l'exposition "Wiphala- Identity and Conflict " (20122013), qui s'articulait autour d'une nouvelle interprétation de l'un des objets des collections andines du musée ${ }^{47}$. Soulignons cependant tout particulièrement ici le projet « Advantage Göteborg " en $2005^{48}$, dont les résultats furent exposés au sein de l'exposition " HorizonsVoices from a Global Africa ». Au sein de ce

48 Pour une analyse de ce projet, voir RINCON, Laurella. "Visiteurs d'origine immigrée et réinterprétation des collections au Världkulturmuseet de Göteborg ». Culture et Musées. $\mathrm{n}^{\circ}$ 6, 2005, p. 111-131. 
dernier, le musée fit participer une trentaine de personnes provenant de la corne de l'Afrique (Éthiopie, Érythrée, Somalie) et résidant à Göteborg, en les invitant à travailler avec les collections en vue de créer des mémoires personnelles et individuelles à partir des objets sélectionnés. Ce projet voulait ainsi explorer la manière dont l'accès à la visibilité institutionnelle combiné à la prise en compte de la réalité mouvante des identités pouvait contribuer à enrichir les spectres d'interprétation de la collection. Par ailleurs, au travers de cette participation, le but de ce projet était de briser les barrières à l'accès au marché du travail par l'augmentation du pouvoir individuel et de la qualification de ces personnes, tout en favorisant leur estime de soi au travers des collections du musée utilisées comme instruments d'habilitation. Cette perspective se trouve bien en phase avec les théories britanniques portant sur le rôle social du musée, étudiées par certains des membres de l'institution suédoise, censé " fournir un espace de tolérance où des problématiques contemporaines difficiles peuvent être explorées en sécurité et dans un esprit de dialogue ${ }^{49} "$.

Cependant, alors que ce projet muséal constituait un ensemble particulièrement cohérent quant à l'adéquation entre sa mission et les actions menées, ce musée connut une phase « d'essoufflement ", à partir de 2010, à la suite d'une grande incertitude quant au futur de l'institution. À partir de cette époque, bon nombre des membres de l'équipe qui participèrent à la création du musée et à son projet initial abandonnèrent progressivement l'institution. En outre, un changement politique à l'échelle nationale se produisit avec l'ascension au pouvoir de la droite conservatrice du parti des Démocrates suédois, après près d'un quart de siècle de gouvernement socio-démocrate. Ce parti, qui a progressivement repris le pouvoir depuis 2009, et tout particulièrement à partir de son entrée au parlement national après les élections de 2010, abandonna progressivement son discours optimiste sur la diversité culturelle en prônant le retour aux valeurs nationalistes. Dans ce contexte, après une phase de relative indépendance de l'institution de Göteborg au sein du consortium national, la direction des musées nationaux de la culture du monde fut rescindée en 2011 en une entité beaucoup plus centralisée tant politiquement que géographiquement (à Stockholm), caractérisée par la volonté de retravailler à partir de ses collections.

\section{Vers la fin d'un modèle?}

De par cet exemple, il convient de se demander si ces rénovations multiculturelles des musées ethnologiques ne se situeraient pas actuellement dans une crise de sens, tel que le laissent présager les nouvelles orientations du musée suédois. Les analyses politiques et médiatiques dominant de nos jours semblent en effet se retourner vers une logique de " choc des cultures $^{50}$ ", alors que la « mort du multiculturalisme " fut annoncée par de nombreuses formations politiques conservatrices depuis 2010 , et ce, avant la vague d'attentats qui toucha la plupart des pays européens.

Devant cette situation, rappelons que certaines des faiblesses de ces rénovations vis-à-vis des fluctuations des discours politiques sont sans aucun doute liées à la nature même de leurs bases conceptuelles. En effet, la représentation des cultures que l'on y trouve semble être plutôt liée à une "muséologie de points de vue ${ }^{51}$ ", particulière aux musées de société, qui ne se centre ni sur l'objet ni sur le savoir, mais plutôt sur le visiteur. Dans ce sens, les expositions répondent non plus à des logiques de recherche visant à une connaissance (comme c'était le cas des musées ethnologiques), mais plutôt à des logiques " communicationnelles ${ }^{52}$ ", articulées autour d'un discours ou d'un message à
49 SANDELL, Richard. Museums, Prejudice and the Reframing of Difference. London: Routledge, 2007, p. 3. 50 Voir HUNGTINGTON, Samuel. The Clash of Civilizations and the Remaking of World Order. New York: Simon \& Schuster, 1996.
51 DAVALLON, Jean. L'exposition à l'œuvre. Stratégies de communication et médiation symbolique. Paris: L'Harmattan, 1999, p. 250.

52 CHAUMIER, Serge. Traité d'expologie. Les écritures de l'exposition. Paris: La Documentation française, 2012, p. 65. 
transmettre au visiteur au travers de la mise en exposition des objets par des professionnels de la muséologie.

Cette approche communicationnelle des rénovations des musées ethnologiques est par ailleurs liée à la volonté de promotion du dialogue des cultures, telle que comprise par les institutions internationales et certains gouvernements nationaux. Cette dernière correspondrait cependant à une vision des contacts entre cultures qui « reproduirait l'idéologie (et donc l'ordre social) qui rend son existence possible ${ }^{53}$ ", souvent bien éloignée des conflits produits par ces rencontres, souvent plus proches des conflits sociaux que culturels. Turgeon indique ainsi que ce cosmopolitisme est implicitement discriminatoire, « se parant de l'esthétique de l'hétérogène, mode de vie élitiste, principalement des classes moyennes, qui aime les emprunts, les mélanges de genres, mais à condition que cette diversité bariolée n'altère pas en profondeur les valeurs persistantes $^{54}$ ». En outre, comme le signalait polites a plus de chance de se donner « dans les plus hautes sphères du spectre culturel, accessible et créé par ceux qui possèdent la mobilité associée à la richesse, l'éducation, l'économie néolibérale et les sociétés démocratiques ${ }^{55}$ ", dont sont issus les membres du musée ainsi qu'une grande partie du public que ces derniers attirent au cours des expositions. Selon cette perspective, l'idée de " discours interculturel " ou de " multiculturalisme " servant de base à ces rénovations correspondrait à une volonté de dépolitisation des rapports sociaux, effaçant au sein de la plupart des musées les conflits que le « multiculturalisme » engendre, tout en renforçant le mécanisme de "reproduction "

53 YAZGI, Nicolas. «L'objet de tous les discours? Ethnographie et muséologie narrative ». GOSETH, MarcOlivier, HAINARD, Jacques et Roland KAERH (dir.). Cent ans d'ethnographie sur la colline de Saint-Nicolas, 1904-2004. Neuchâtel: Musée d'ethnographie, 2005, p. 596.

54 TURGEON. op.cit., p. 196.

55 BAUMAN, op. cit., p. 3 et 86.

56 Voir notamment BOURDIEU, Pierre et Alain DARBEL. L'amour de l'art: les musées et leur public. Paris: Minuit, 1969.

57 GRINDELL, Klas. « When legitimate claims collide: communities, media and dialogue \%. Museum and Society. vol. 9, n 3, 2011, p. 232.

58 DE L'ESTOILE, Benoît. «L'Anthropologie après les musées? ". Ethnologie française, $\mathrm{n}^{\circ} 4$, 2008, p. 669-670.

59 Ibid., p. 670.

60 JAMIN, op.cit., p. 69.

61 STURTEVANT, op.cit. des hiérarchies classes sociales, tel que soulevé par Bourdieu ${ }^{56}$, au travers, cette fois-ci, d'une certaine idée du cosmopolitisme. Comme le reconnaissait Grinell, commissaire du musée suédois, alors que les musées souhaitent incarner des arènes de discussion, invitant le public à un dialogue libre, «il existe une grande différence entre ceux qui se sentent à l'aise dans cette arène $\mathrm{e}^{57}$ \%.

Devant ces fragilités de la base multiculturelle de ces rénovations, afin de trouver un sens dans un contexte marqué par de nouvelles compréhensions du monde, il serait sans doute intéressant pour ces musées de se retourner vers l'anthropologie et les sciences sociales en vue de poser un nouveau regard sur leurs pratiques. En effet, alors que cette institution constituerait pour De L'Estoile l'un des lieux essentiels « où se joue la compréhension du monde contemporain dans sa complexité ${ }^{58}$ ", la présence des anthropologues y jouerait un rôle important alors que ces derniers sont progressivement devenus les spécialistes des " relations entre des personnes qui appartiennent à des mondes différents ${ }^{59}$ ". Au sein de la construction d'un nouveau discours, la fondation d'une ethnomuséologie appliquée permettrait, selon Jamin, de " sortir à la fois de la culpabilité coloniale - que l'existence de ces musées réactive malgré tout -, des atermoiements du postcolonialisme et des soupçons du postmodernisme qui ont condamné nombre d'entre eux à la paralysie ou à l'impuissance ${ }^{60}$ ". Cette recréation des liens entre musée et anthropologie, maintes fois souhaitée depuis les années 1960 et le développement de "l'âge universitaire de l'Anthropologie ${ }^{61}$ ", éloignée du musée, reste sans aucun doute la solution pour que ces musées, fruits d'une longue 
et riche histoire, continuent d'apporter une réponse décomplexée et sereine à la question existentielle de savoir qui nous sommes, et de définir notre place dans un monde toujours plus complexe et interconnecté. 
From the ethnology museum to the multicultural museum.
Stakes and appraisal of a European institutional renovation.

In an era marked by questions of how to respond to immigration and by the highlighting of intercultural dialogue by international institutions, seen as guarantors of social cohesion, many ethnological museums in Europe underwent extensive renovation throughout the 1990s and 2000s. In fact, while their predecessors were immersed in the profound crisis of decolonization, because of their collections, these renovations were seen as favoured instruments to promote multicultural values and acceptance of the cultural diversity engendered by immigration into Western societies.

More than a decade after the beginning of this spate of renovations which spread all the way across Europe, this article hopes to provide a reflexive assessment of these transformations. Using a process of abstraction, it will first address the museological strategies instituted within the museums and the new methodologies found there, while identifying their common features. Furthermore, through analysis of the methodolgies and strategies established by the Museum of World Culture in Gothenburg (Sweden), this article will attempt to highlight the principle challenges encountered in these renovations.

In conclusion, the article will attempt to explain the existence of the complexities, by characterizing the renovations as a "museology of points of view," whose foundation is formed by the spatial installation

of a message defined outside of the museum, and particularly by political and supranational interests. While this characteristic makes the renovations particularly vulnerable to current changes in understanding of the issues of cultural diversity, the article will finish with the wish to see new bonds forged between these museums and anthropology, with a view to strengthening their theoretical content and guaranteeing their future. 